\title{
Formulation of the Post-Newtonian Equations of Motion of the Restricted Three Body Problem
}

\author{
Fawzy A. Abd El-Salam ${ }^{1}$, Sobhy Abd El-Bar ${ }^{2}$ \\ ${ }^{1}$ Department of Astronomy, Faculty of Science, Cairo University, Cairo, Egypt \\ ${ }^{2}$ Department of Mathematics, Faculty of Science, Tanta University, Tanta, Egypt \\ E-mail:f.a.abdelsalam@gmail.com,fawzy_zaher@yahoo.co.uk \\ Received May 15, 2010; revised November 10, 2010; accepted November 14, 2010
}

\begin{abstract}
In the present work the geodesic equation represents the equations of motion of the particles along the geodesics is derived. The deviation of the curved space-time metric tensor from that of the Minkowski tensor is considered as a perturbation. The quantities is expanded in powers of $c^{-2}$. The equations of motion of the relativistic three body problem in the PN formalism are obtained.
\end{abstract}

Keywords: Post Newtonian Approximation, Geodesic Equation, Restricted Three Body Problem

\section{Introduction}

The Three body problem concerns with the motion of a small particle of negligible mass moving under the gravitational influence of two massive objects $m_{1}$ and $m_{2}$. It's history began with Euler and Lagrange continues with Jacobi [1], Hill [2], Poincaré [3], and Birkhoff [4]. In 1772, Euler [5] first introduced a synodic (rotating) coordinate system, the use of which led to an integral of the equations of motion, known today as the Jacobian integral. Euler himself did not discover the Jacobia integral which was first given by Jacobi [1] who, as Wintner remarks, "rediscovered" the synodic system. The actual situation is somewhat complex since Jacobia published his integral in a sideral (fixed) system in which its significance is definitely less than in the synodic system.

Many authors hope to investigate the relativistic effects in this problem. But unfortunately, the Einstein field equations are nonlinear, and therefore cannot in general be solved exactly. By imposing the symmetry requirements of time independence and spatial isotropy we are able to find one useful exact solution, the Schwarzschild metric, but we cannot actually make use of the full content of this solution, because in fact the solar system is not static and isotropic.

Indeed, the Newtonian effects of the planet's gravitational fields are an order of magnitude greater than the first corrections due to general relativity, and completely swamp the higher corrections that are in principle provided by the exact Schwarzschild solution. It is worth noting to highlight some important articles in this field.

Krefetz [6] computed the post-Newtonian deviations of the triangular Lagrangian points from their classical positions in a fixed frame of reference for the first time, but without explicitly stating the equations of motion. Contopoulos [7] treated the relativistic (RTBP) in rotating coordinates. He derived the Lagrangian of the system and the deviations of the triangular points as well. Weinberg [8] calculated the components of the metric tensor by using the post-Newtonian approximation in order to obtain the (RTBP) problem equations of motion. Soffel [9] obtained The angular frequency $\omega$ of the rotating frame for the relativistic two-body problem. Brumberg $[10,11]$ studied the problem in more details and collected most of the important results on relativistic celestial mechanics. He did not obtain only the equations of motion for the general problem of three bodies but also deduced the equations of motion for the restricted problem of three bodies. Bhatnagar and Hallan [12] studied the existence stability of the triangular points $L_{4,5}$ in the relativistic (RTBP), they concluded that $L_{4,5}$ are always unstable in the whole range $0 \leq \mu \leq 0.5$ in contrast to the previous results of the classical restricted three-body problem where they are stable for $0 \leq \mu<\mu_{0}$ where $\mu$ is the mass ratio and $\mu_{0}=0.03852$. Lucas [13] found that the difference between Newtonian and post-Newtonian trajectories for the restricted three-body problem is greater for chaotic trajectories than it is for trajectories that are not chaotic. Finally, the possibility of using this Chaotic Amplification Effect as a novel test of general 
relativity is discussed.

\section{Expansion Dimensionless Parameter}

In the presence of weak gravitational fields and small velocities, the physics of general relativity should become Newtonian. This suggest an expansion of the metric, Christoffel symbols, other tensors and the field equations in powers of small parameter, similar to the perturbation expansions of classical and quantum mechanics.

The nature and value of the expansion parameter is determined by the system at hand. But in general we can characterize a self-gravitating system by a characteristic mass $M$, characteristic length $L$ and a characteristic time $T$. The mass could be the mass of the principal body in the system. The length could be its physical radius, and the time could be the time it takes an object to traverse the length scale. Each of these quantities set a scale for its particular dimension, and from them we can establish the dimensionless quantities.

In a bound system where motion is periodic, the Virial theorem says (for a nonrelativistic velocities) that

$$
\frac{1}{2} m v^{2}=-\frac{1}{2} V
$$

or, for a Newtonian gravitational potential $\frac{G m M}{r}$

$$
v^{2}=-\frac{G M}{r}
$$

Dividing both sides by the $c^{2}$, where $c$ is the velocity of light yields

$$
\frac{v^{2}}{c^{2}}=-\frac{G M}{c^{2} r}
$$

This very simple equation enables us to identify the expansion parameter as

$$
\varepsilon^{2}=\left(\frac{L}{c^{2} T}\right)^{2}=-\frac{G M}{c^{2} L}
$$

In the following we will set $G=c=L=1$, and have

$$
\varepsilon=T^{-1} \quad \varepsilon^{2}=M
$$

As an example, we can compute the parameter $\varepsilon$ for the Sun, taking its radius as the distance scale.

$$
\varepsilon=\sqrt{\frac{1.477 \times 10^{3}}{6.9598 \times 10^{8}}}=1.4586 \times 10^{-3}
$$

What we need then is not to find more exact solutions, but rather to develop some systematic approximation method that will not rely on any assumed symmetry properties of the system.

There are two such methods that have been particular- ly useful they are called the Post-Newtonian approximation (PN) and the weak field approximation. The first is adapted to a system of slowly moving particles bound together by gravitational forces. The second method treats the fields in a lower order of approximation but does not assume that the matter moves non-relativistically. A test particle in a circular orbit of radius $r$ about a central mass $M$ will have velocity $v$ given in Newtonian mechanics by the exact formula $v^{2}=(G M) / r$.

The PN approximation may be described as a method for obtaining the motions of the system to one higher power of the small parameters $(G M) / r$ and $v^{2}$ than given by Newtonian mechanics. It is sometimes referred to as an expansion in inverse powers of the speed of light. We prefer to say that our expansion parameter is $c^{-2}$, note that geometric units will not be used, so that $G \neq 1$, and $c \neq 1$. We now proceed to find the equations of motion of the relativistic three body problem in the PN formalism, or more precisely the equation of the RTBP.

\section{The Geodesic Equation}

According to the theory of general relativity a particle moving under the influence of gravity follows a Geodesic in a four dimensional space called the space-time manifold. The path that it follows is called a geodesic. Let the coordinate of this manifold be as follows: $x_{0}=-c t$, $x_{1}=x, x_{2}=y$ and $x_{3}=z$. Consider a particle moving along a geodesic $x^{\mu}(s)$ in the space-time manifold, where $s$ is the arc length. A straight line is defined as any path in which its tangent vectors all points in the same direction. Let the tangent to the curve $x^{\mu}$ (s) be defined

$$
A^{\mu}=\frac{d x^{\mu}(s)}{d s}
$$

The magnitude of this tangent vector is obtained from the tensor algebra as;

$$
A=\left(g_{\mu \gamma} A^{\mu} A^{\gamma}\right)^{\frac{1}{2}}
$$

where $g_{\mu \gamma}$ is the metric tensor, which can be obtained from

$$
d s^{2}=g_{\mu \gamma} d x^{\mu} d x^{\gamma}
$$

substituting into Equation (2) yields

$$
A= \pm 1
$$

which means that the tangent vectors all have a constant length, usually called the unit tangent to the curve. This property leads to (when differentiating)

$$
\frac{d A^{\mu}}{d s}=0
$$


If a unit tangent $A^{\mu}$ is parallel displaced from a point $p$ to a point $p^{\prime}$ one can easily get

$$
\delta A^{\mu}=-\Gamma_{\gamma \lambda}^{\mu} A^{\gamma} d x^{\lambda}
$$

where $\Gamma_{\gamma \lambda}^{\mu}$ is the affine connection, to be defined later. $\delta A^{\mu}$, using the elementary mathematics, can be written as

$$
\delta A^{\mu}=\left(A^{\mu}\right)_{p^{\prime}}-\left(A^{\mu}\right)_{p}=\frac{\partial A^{\mu}}{\partial s} d s
$$

substituting from Equation (5) into Equation (6) yields

$$
c \frac{d A^{\mu}}{d s}+\Gamma_{\gamma \lambda}^{\mu} A^{\gamma} \frac{d x^{\lambda}}{d s}=0
$$

this equation merely represents a division of $d A^{\mu}$ by $d s$ and not to be confused with Equation (4). Substituting from Equation (1) into Equation (7) yields directly the geodesic equation. In fact that the geodesic equation represents the equations of motion of the particles along the geodesics

$$
\frac{d^{2} x^{\mu}}{d s^{2}}+\Gamma_{\gamma \lambda}^{\mu} \frac{d x^{\gamma}}{d s} \frac{d x^{\lambda}}{d s}=0
$$

Since the proper time $\tau$ is proportional to the arclength these equations can be written as

$$
\frac{d^{2} x^{\mu}}{d \tau^{2}}+\Gamma_{\gamma \lambda}^{\mu} \frac{d x^{\gamma}}{d \tau} \frac{d x^{\lambda}}{d \tau}=0
$$

where the affine connection $\Gamma_{\gamma \lambda}^{\mu}$ is given by

$$
\Gamma_{\gamma \lambda}^{\mu}=\frac{1}{2} g^{\mu p}\left\{\frac{\partial g_{p \gamma}}{\partial x^{\lambda}}+\frac{\partial g_{p \lambda}}{\partial x^{\gamma}}-\frac{\partial g_{\gamma \lambda}}{\partial x^{p}}\right\}
$$

Now the accelerations can be computed using Equation (9) as

$$
\begin{aligned}
\frac{d^{2} x^{i}}{d t^{2}} & =\frac{d}{d t}\left[\frac{d x^{i}}{d \tau} \frac{d \tau}{d t}\right] \\
& =\frac{d^{2} x^{i}}{d \tau^{2}}\left(\frac{d \tau}{d t}\right)^{2}+\frac{d x^{i}}{d \tau} \frac{d}{d t}\left(\frac{d t}{d \tau}\right)^{-1} \\
& =\frac{d^{2} x^{i}}{d \tau^{2}}\left(\frac{d \tau}{d t}\right)^{2}-\frac{d x^{i}}{d \tau}\left(\frac{d t}{d \tau}\right)^{-2} \frac{d}{d t}\left(\frac{d t}{d \tau}\right) \\
& =\frac{d^{2} x^{i}}{d \tau^{2}}\left(\frac{d t}{d \tau}\right)^{-2}-\frac{d x^{i}}{d \tau}\left(\frac{d t}{d \tau}\right)^{-2} \times\left(\frac{d}{d \tau} \frac{d \tau}{d t}\left(\frac{d t}{d \tau}\right)\right) \\
& =\frac{d^{2} x^{i}}{d \tau^{2}}\left(\frac{d t}{d \tau}\right)^{-2}-\frac{d x^{i}}{d \tau}\left(\frac{d t}{d \tau}\right)^{-3} \frac{d^{2} \tau}{d t^{2}} \frac{d^{2} x^{i}}{d t^{2}} \\
& =-\Gamma_{\gamma \lambda}^{i} \frac{d x^{\gamma}}{d t} \frac{d x^{\lambda}}{d t}+\Gamma_{\gamma \lambda}^{0} \frac{d x^{\gamma}}{d t} \frac{d x^{\lambda}}{d t} \frac{d x^{i}}{d t}
\end{aligned}
$$

where we made use the geodesic Equation (9) for the inboxed terms.

Remark: We now perform the sums over the dummy indices, namely $\gamma$, and $\lambda$ to separate the time and space indexed term,

$$
\begin{aligned}
\frac{d^{2} x^{i}}{d t^{2}}= & -\Gamma_{00}^{i}-2 \Gamma_{0 \lambda}^{i} \frac{d x^{\lambda}}{d t}-\Gamma_{j k}^{i} \frac{d x^{j}}{d t} \frac{d x^{k}}{d t} \frac{d x^{i}}{d t} \\
& +\left\{\Gamma_{00}^{0}+2 \Gamma_{0 j}^{i} \frac{d x^{j}}{d t}+\Gamma_{j k}^{i} \frac{d x^{j}}{d t} \frac{d x^{k}}{d t}\right\} \frac{d x^{i}}{d t}
\end{aligned}
$$

In the Newtonian limit we treat all velocities as vanishingly small, and so have to zeroth Newtonian order

$$
\begin{aligned}
\frac{d^{2} x^{i}}{d t^{2}} & \approx-\Gamma_{00}^{i} \\
& \approx \frac{1}{2} \frac{\partial g_{00}}{\partial x^{i}}=\frac{1}{2} \frac{\partial}{\partial x^{i}}\left(1-\frac{G M}{c^{2} r}\right) \\
& =\frac{G M}{c^{2} r^{2}} \approx O\left(c^{-2}\right)
\end{aligned}
$$

\section{The Metric Tensor}

Since a body of a mass $m_{b}$ tends to curve space-time, the metric of the space-time will deviate from that of the Minkowski tensor which represents the flat space. But assuming, without loss of the accuracy required, that the mass of the body is so small, so that the departure from the Minkowski tensor will be in powers of $c^{-1}$, or in other words the effect on the space-time can be considered as a perturbation to the metric of the flat space, i.e.

$$
g_{\mu \gamma}=\eta_{\mu \gamma}+h_{\mu \gamma} \quad\left|h_{\mu \gamma}\right| \text { is so small }
$$

where $\eta_{\mu \gamma}$, the metric of the flat space-time, is given, in matrix representation, by

$$
\eta=\left(\begin{array}{cccc}
-1 & 0 & 0 & 0 \\
0 & 1 & 0 & 0 \\
0 & 0 & 1 & 0 \\
0 & 0 & 0 & 1
\end{array}\right)
$$

Using the fact that the velocity of a test particle of mass $m_{p}$ relative to the body is $v \ll c$, and assuming $m_{p} / m_{b} \approx 0$, so that the effects on space-time originating from the particle are negligible, the metric tensor of the perturbed flat space-time can generally be described by

$$
\left.\begin{array}{l}
g_{00}=1+h_{00} \\
g_{i j}=\delta_{i j}+h_{i j} \\
g_{i 0}=h_{i 0}
\end{array}\right\}
$$

We see that the metric tensor is no longer diagonal. We would like to find the corrections to the metric ten- 
sor, induced by the theory of general relativity, that is, to determine $h_{00}$ to order $O\left(c^{-6}\right)$ and $h_{0 j}$ to order $O\left(c^{-5}\right)$, by solving the Einstein field equations for $h_{00}$ and $h_{0 j}$. Our objective in using the PN approximation will be to compute $\left(d^{2} x^{i}\right) /\left(d t^{2}\right)$ to order $c^{-4}$. Since the $g_{i j}$ are expected to include powers of $(G M) / r$, the partial derivatives of the $g_{i j}$ are significantly smaller than $g_{i j}$ themselves.

The components of the metric tensor are assumed expandable as

$$
\left.\begin{array}{c}
g_{00}=-1+\stackrel{2}{g}_{00}+\stackrel{4}{g}_{00}+ \\
g_{i j}=\delta_{i j}+\stackrel{4}{g}_{i j}+\stackrel{4}{g}_{i j}+\cdots \\
\stackrel{3}{g}_{i 0}+{ }^{5} g_{i 0}+\cdots
\end{array}\right\}
$$

The symbol ${ }_{\mu \gamma}^{N}$ denoting the term in $g_{\mu \gamma}$ of order $1 / c^{N}$. Odd powers of $c \frac{1}{c}$ occur in $g_{i 0}$ because $g_{i 0}$ must change sign under the time-reversal transformation $t \rightarrow-t$.

The inverse of the metric tensor is defined by the equations

$$
\left.\begin{array}{c}
g^{i \mu} g_{0 \mu}=g^{i 0} g_{00}+g^{i j} g_{j 0}=0, \\
g^{0 \mu} g_{0 \mu}=g^{00} g_{00}+g^{0 i} g_{o i}=1, \\
g^{i \mu} g_{j \mu}=g^{i 0} g_{j 0}+g^{i k} g_{j k}=\delta_{i j} .
\end{array}\right\}
$$

We expect that

$$
\left.\begin{array}{l}
g^{00}=-1+\stackrel{2}{g}^{00}+\stackrel{4}{g}^{00}+\cdots \\
g^{i j}=\delta_{i j}+\stackrel{4}{g}^{i j}+\stackrel{4}{g}^{i j}+\cdots \\
g^{i 0}=\stackrel{3}{g}^{i 0}+\stackrel{5}{g}^{i 0}+\cdots
\end{array}\right\}
$$

Using these expansions into (15) we find

$$
\left.\begin{array}{c}
\stackrel{2}{g}^{00}=-\stackrel{2}{g}_{00} \\
2^{i j}=-\stackrel{2}{g}_{i j}, \\
\stackrel{3}{g}^{i 0}=-\stackrel{3}{g}_{i 0}
\end{array}\right\}
$$

The affine connection may now be obtained from the familiar formula

$$
\Gamma_{\gamma \lambda}^{\mu}=\frac{1}{2} g^{\mu p}\left\{\frac{\partial g_{p \gamma}}{\partial x^{\lambda}}+\frac{\partial g_{p \lambda}}{\partial x^{\gamma}}-\frac{\partial g_{\gamma \lambda}}{\partial x^{p}}\right\}
$$

Using (15), (16) and (17) we find that $\Gamma_{00}^{i}, \Gamma_{j k}^{i}$ and $\Gamma_{0 i}^{o}$ have the expansions

$$
\Gamma_{\gamma \lambda}^{\mu}=\stackrel{2}{\Gamma}_{\gamma \lambda}^{\mu}+\stackrel{4}{\Gamma}{ }_{\gamma \lambda}^{\mu}+\cdots
$$

While the components $\Gamma_{0 j}^{i}, \Gamma_{00}^{0}$ and $\Gamma_{i j}^{0}$ have the expansions

$$
\Gamma_{\gamma \lambda}^{\mu}=\stackrel{3}{\Gamma}_{\gamma \lambda}^{\mu}+\stackrel{3}{\Gamma}_{\gamma \lambda}^{\mu}+\cdots
$$

The symbol $\Gamma_{\gamma \lambda}^{N}$, denoting the non vanishing terms in $\Gamma_{\gamma \lambda}^{\mu}$ of order $1 / c^{N}$, are given by

$$
\begin{aligned}
& \stackrel{2}{\Gamma}_{00}^{i}=-\frac{1}{2} \frac{\partial \stackrel{2}{g}_{00}}{\partial x^{i}} \\
& \stackrel{4}{\Gamma}_{00}^{i}=-\frac{1}{2} \frac{\partial \stackrel{4}{g}_{00}}{\partial x^{i}}+\frac{\partial \stackrel{3}{g}_{i 0}}{\partial t}+\frac{1}{2} \stackrel{2}{g}_{i j} \frac{\partial \stackrel{2}{g}_{00}}{\partial x^{j}} \\
& \stackrel{3}{\Gamma}_{0 j}^{i}=\frac{1}{2}\left\{\frac{\partial \stackrel{3}{g}_{i 0}}{\partial x^{j}}+\frac{\partial \stackrel{2}{g}_{i j}}{\partial t}-\frac{\partial \stackrel{3}{g}_{j 0}}{\partial x^{i}}\right\} \\
& \stackrel{2}{\Gamma}_{j k}^{i}=\frac{1}{2}\left\{\frac{\partial \stackrel{2}{g}_{i j}}{\partial x^{k}}+\frac{\partial \stackrel{2}{g}_{i k}}{\partial x^{j}}-\frac{\partial \stackrel{2}{g}_{j k}}{\partial x^{i}}\right\} \\
& \stackrel{3}{\Gamma}_{00}^{0}=-\frac{1}{2} \frac{\partial \stackrel{2}{g}_{00}}{\partial t} \\
& \stackrel{2}{\Gamma}_{0 i}^{0}=-\frac{1}{2} \frac{\partial \stackrel{2}{g}_{00}}{\partial x^{i}}
\end{aligned}
$$

\section{Einstein Field Equations}

We are now ready to compute the metric tensor components $g_{i j}$ up to the different orders appeared in Equations (15) and (16). To do that, the Einstein field equations will be used in the following form.

$$
R_{\mu \gamma}=-\frac{8 \pi G}{c^{4}}\left(T_{\mu \gamma}-\frac{1}{2} g_{\mu \gamma} T_{\lambda}^{\lambda}\right)
$$

where $R_{\mu \gamma}$ is the Ricci tensor and $T_{\mu \gamma}$ is the stressenergy-momentum tensor. Note that Greek indices range from 0 to 3 while roman indices range from 1 to 3 .

\section{The Ricci Tensor $R_{\mu \kappa}$}

The Ricci tensor is defined by

$$
\begin{aligned}
R_{\mu \kappa}= & \frac{\partial}{\partial x^{\kappa}} \Gamma_{\mu \lambda}^{\lambda}-\frac{\partial}{\partial x^{\lambda}} \Gamma_{\mu \kappa}^{\lambda} \\
& +\Gamma_{\mu \kappa}^{\alpha} \Gamma_{\kappa \alpha}^{\lambda}-\Gamma_{\mu \kappa}^{\alpha} \Gamma_{\alpha \lambda}^{\lambda}
\end{aligned}
$$

we find that the components of $R_{\mu \kappa}$ have the expansions, 


$$
\left.\begin{array}{rl}
R_{00} & =\stackrel{2}{R}_{00}+\stackrel{4}{R}_{00}+\cdots \\
& 3 \\
R_{i 0} & =\stackrel{5}{R}_{i 0}+R_{i 0}+\cdots \\
2 & 4 \\
R_{i j} & \stackrel{4}{R}_{i j}+R_{i j}+\cdots
\end{array}\right\}
$$

where $\stackrel{N}{R_{i j}}$ denotes the term in $R_{i j}$ of order $1 / c^{N}$. The terms that we can calculate from the known terms in the affine connection are

$$
\begin{aligned}
\stackrel{2}{R}_{00}= & -\frac{\partial}{\partial x^{i}} \stackrel{2}{\Gamma}_{00}^{i} \\
\stackrel{4}{R}_{00}= & \frac{\partial}{\partial t} \stackrel{3}{\Gamma}_{i 0}^{i}-\frac{\partial}{\partial x^{i}} \stackrel{4}{\Gamma}_{00}^{i} \\
& +\stackrel{2}{\Gamma}_{0 i}^{0} \stackrel{2}{\Gamma}_{00}^{i}-\stackrel{2}{\Gamma}_{00}^{i} \stackrel{2}{\Gamma}_{i j}^{j} \\
\stackrel{3}{R}_{i 0}= & \frac{\partial}{\partial t} \stackrel{2}{\Gamma}_{i j}^{j}-\frac{\partial}{\partial x^{j}} \stackrel{3}{\Gamma}_{0 i}^{j} \\
\stackrel{2}{R}_{i j}= & \frac{\partial}{\partial x^{j}} \stackrel{2}{\Gamma}_{i 0}^{0}+\frac{\partial}{\partial x^{j}} \stackrel{2}{\Gamma}_{i k}^{k} \\
& -\frac{\partial}{\partial x^{k}} \stackrel{2}{\Gamma}_{i j}^{k}
\end{aligned}
$$

Using Equation (22), we obtain

$$
\begin{aligned}
& \stackrel{2}{R_{00}}=\frac{1}{2} \nabla^{2} \stackrel{2}{g}_{00} \\
& \stackrel{4}{R}_{00}=\frac{1}{2} \frac{\partial^{2} \stackrel{2}{g}_{i j}}{\partial t^{2}}-\frac{\partial^{2} \stackrel{3}{g}_{i 0}}{\partial x^{i} \partial t}+\frac{1}{2} \nabla^{2} \stackrel{4}{g}_{00} \\
& -\frac{1}{2} \stackrel{2}{g}_{i j} \frac{\partial^{2} \stackrel{2}{g}_{00}}{\partial x^{i} \partial x^{j}}-\frac{1}{2}\left(\frac{\partial \stackrel{2}{g}_{i j}}{\partial x^{j}}\right)\left(\frac{\partial \stackrel{2}{g}_{00}}{\partial x^{i}}\right) \\
& +\frac{1}{4}\left(\frac{\partial \stackrel{2}{g}_{0 i}}{\partial x^{i}}\right)\left(\frac{\partial \stackrel{2}{g}_{i 0}}{\partial x^{i}}\right)+\frac{1}{4}\left(\frac{\partial \stackrel{2}{g}_{i 0}}{\partial x^{i}}\right)\left(\frac{\partial \stackrel{2}{g}_{i j}}{\partial x^{i}}\right) \\
& \stackrel{3}{R}_{i 0}=\frac{1}{2} \frac{\partial^{2} \stackrel{2}{g}_{i j}}{\partial x^{i} \partial t}-\frac{1}{2} \frac{\partial^{2} \stackrel{3}{g}_{j 0}}{\partial x^{i} \partial x^{j}}-\frac{1}{2} \frac{\partial^{2} \stackrel{2}{g}_{i j}}{\partial x^{j} \partial t}+\frac{1}{2} \nabla^{2} \stackrel{3}{g}_{i 0} \\
& \stackrel{2}{R}_{i j}=-\frac{1}{2} \frac{\partial^{2} \stackrel{2}{g}_{00}}{\partial x^{i} \partial x^{j}}+\frac{1}{2} \frac{\partial^{2} \stackrel{2}{g}_{k k}}{\partial x^{i} \partial x^{j}} \\
& -\frac{1}{2} \frac{\partial^{2} \stackrel{2}{g}_{i k}}{\partial x^{k} \partial x^{j}}-\frac{1}{2} \frac{\partial^{2} \stackrel{2}{g}_{k j}}{\partial x^{k} \partial x^{i}}+\frac{1}{2} \nabla^{2} \stackrel{2}{g}_{i j}
\end{aligned}
$$

A tremendous simplification can be achieved at this point by choosing a suitable coordinates system.

It is always possible to define the $x^{\mu}$ so that they obey the harmonic coordinate conditions, Weinberg [8]

$$
g^{\mu \gamma} \Gamma_{\mu \gamma}^{\lambda}=0
$$

Using Equations (16), (17), (19) and (21) we find that the vanishing of the third-order term in $g^{\mu \gamma} \Gamma_{\mu \gamma}^{0}$ gives

$$
\frac{1}{2} \frac{\partial \stackrel{2}{g}_{00}}{\partial t}-\frac{\partial \stackrel{2}{g}_{0 i}}{\partial x^{i}}+\frac{1}{2} \frac{\partial \stackrel{2}{g}_{i i}}{\partial t}=0
$$

While the vanishing of the second-order term in $g^{\mu \gamma} \Gamma_{\mu \gamma}^{i}$ gives

$$
\frac{1}{2} \frac{\partial \stackrel{2}{g}_{00}}{\partial x^{i}}+\frac{\partial \stackrel{3}{g}_{i j}}{\partial x^{j}}-\frac{1}{2} \frac{\partial \stackrel{2}{g}_{j j}}{\partial x^{i}}=0
$$

It follows that

$$
\left.\begin{array}{l}
\frac{1}{2} \frac{\partial^{2} \stackrel{2}{g}_{i i}}{\partial t^{2}}-\frac{\partial^{2} \stackrel{2}{g}_{i 0}}{\partial t \partial x^{i}}+\frac{1}{2} \frac{\partial^{2} \stackrel{2}{g}_{00}}{\partial t^{2}}=0 \\
\frac{1}{2} \frac{\partial \stackrel{4}{g}_{i i}}{\partial t \partial x^{j}}-\frac{\partial^{2} \stackrel{2}{g}_{i 0}}{\partial x^{i} \partial x^{j}}-\frac{\partial^{2} \stackrel{2}{g}_{i j}}{\partial x^{i} \partial t}=0 \\
\frac{\partial^{2} \stackrel{2}{g}_{i j}}{\partial x^{k} \partial x^{j}}+\frac{\partial^{2} \stackrel{2}{g}_{k j}}{\partial x^{j} \partial x^{i}} \\
-\frac{\partial^{2} \stackrel{2}{g}_{i j}}{\partial x^{i} \partial x^{k}}+\frac{\partial^{2} \stackrel{2}{g}_{00}}{\partial x^{i} \partial x^{k}}=0
\end{array}\right\}
$$

So the simplified formulas for the Ricci tensor will be in the form

$$
\left.\begin{array}{l}
\stackrel{2}{R}_{00}=\frac{1}{2} \nabla^{2} \stackrel{2}{g}_{00} \\
\stackrel{4}{R}_{00}=\frac{1}{2} \nabla^{2} \stackrel{4}{g}_{00}-\frac{1}{2} \frac{\partial^{2} \stackrel{2}{g}_{00}}{\partial t^{2}}+\frac{1}{2} \nabla^{2} \stackrel{4}{g}_{00} \\
\quad-\frac{1}{2} \stackrel{2}{g}_{i j} \frac{\partial^{2} \stackrel{2}{g}_{00}}{\partial x^{i} \partial x^{j}}+\frac{1}{2} \nabla^{2} \stackrel{2}{g}_{00} \\
\left(\begin{array}{l}
3 \\
R_{0 i}
\end{array}\right)^{3}=\frac{1}{2} \nabla^{2}\left(\begin{array}{l}
3 \\
g_{i 0}
\end{array}\right)^{3} \\
\left(\begin{array}{l}
2 \\
R_{i j}
\end{array}\right)^{2}=\frac{1}{2} \nabla^{2}\left(\begin{array}{l}
2 \\
g_{i j}
\end{array}\right)^{2} .
\end{array}\right\}
$$

Substituting into Equation (24), yields

$$
\left.\begin{array}{rl}
R_{00}= & \frac{1}{2} \nabla^{2} \stackrel{2}{g}_{00}+\frac{1}{2} \nabla^{2} \stackrel{4}{g}_{00} \\
& -\frac{1}{2} \stackrel{2}{g}_{i j} \frac{\partial^{2} \stackrel{2}{g}_{00}}{\partial x^{i} \partial x^{j}}+\frac{1}{2} \nabla^{2} \stackrel{2}{g}_{00} \\
R_{0 i}= & \frac{1}{2} \nabla^{2} \stackrel{3}{g}_{i 0} \\
R_{i j}= & \frac{1}{2} \nabla^{2} \stackrel{2}{g}_{i j}
\end{array}\right\}
$$




\section{The Energy-Momentum Tensor $T_{\mu \gamma}$}

It is assumed that, it is possible to describe the matter of a system as a perfect fluid, i.e. an isotropic fluid with a diagonal energy-momentum tensor (no shear stress), in the rest frame of the system. This energy-momentum tensor which is required in the field equation can be written as

$$
T^{\mu \gamma}=\frac{1}{\sqrt{g}} \sum_{n=1}^{2} M_{n} \frac{d x_{n}^{\mu}}{d t} \frac{d x_{n}^{\gamma}}{d t}\left(\frac{d \tau_{n}}{d t}\right)^{-1} \delta^{3}\left(x-x_{n}\right)
$$

where $g$ is the determinant of the metric tensor and $M_{n}, x_{n}^{\mu}$ and $\tau_{n}$ are the mass, space-time position, and proper time for the two massive objects. The expression is simplified by calculating the energy-momentum tensor in the rotating frame, in which the velocity of the primaries is zero. The nonvanishing components of the energymomentum tensor are

$$
\begin{aligned}
T^{00}= & \frac{c^{2}}{\sqrt{g}}\left\{M_{1}\left(\frac{d t}{d \tau_{1}}\right) \delta^{3}\left(x-x_{1}\right)\right. \\
& \left.+M_{2}\left(\frac{d t}{d \tau_{2}}\right) \delta^{3}\left(x-x_{2}\right)\right\}
\end{aligned}
$$

We expect that $T^{00}, T^{i 0}$ and $T^{i j}$ will have the expansions

$$
\left.\begin{array}{l}
T^{00}=\stackrel{0}{T}^{00}+\stackrel{2}{T}^{00}+\cdots \\
T^{i 0}=\stackrel{1}{T}^{i 0}+\stackrel{3}{T}^{i 0}+\cdots \\
T^{i j}=\stackrel{2}{T}^{i j}+\stackrel{4}{T}^{i j}+\cdots
\end{array}\right\}
$$

where $T^{N}{ }^{\mu \gamma}$ denotes the term in $T^{\mu \gamma}$ of order $1 / c^{N}$. In particular $\stackrel{0}{T}^{00}$ is the density of rest-mass, while $\stackrel{2}{T}^{00}$ is the non-relativistic part of the energy density.

What we need now is

$$
S_{\mu \gamma}=T_{\mu \gamma}-\frac{1}{2} g_{\mu \gamma} T_{\lambda}^{\lambda}
$$

So that Equation (35) gives

$$
\left.\begin{array}{l}
S_{00}=\stackrel{0}{S}_{00}+\stackrel{2}{S}_{00}+\cdots \\
S_{i 0}=\stackrel{3}{S}_{i 0}+\stackrel{3}{S}_{i 0}+\cdots \\
S_{i j}=\stackrel{0}{S}_{i j}+\stackrel{2}{S}_{i j}+\cdots
\end{array}\right\}
$$

where $\stackrel{N}{S}_{\mu \gamma}$ denotes the term in $S_{\mu \gamma}$ of order $1 / c^{N}$ In particular

$$
\left.\begin{array}{l}
\stackrel{0}{S_{00}}=\frac{1}{2} \stackrel{0}{T}^{00} \\
\stackrel{0}{S_{00}}=\frac{1}{2}\left[\stackrel{2}{T}^{00}-2 \stackrel{2}{g}_{00} \stackrel{0}{T}^{00}+\stackrel{2}{T}^{i j}\right] \\
\stackrel{1}{S_{i 0}}=-\stackrel{1}{T^{0 i}} \\
\stackrel{0}{S_{i j}}=\frac{1}{2} \delta_{i j} \stackrel{0}{T}^{00}
\end{array}\right\}
$$

Using Equations (31) and (37) in the field Equation (22), we find that the field equations in harmonic coordinates are indeed consistent with the expansions we have been using, and give

$$
\left.\begin{array}{rl}
\nabla^{2} \stackrel{2}{g}_{00} & =-\frac{8 \pi G}{c^{4}} \stackrel{0}{T} 00 \\
\nabla^{2} \stackrel{4}{g}_{00} & =g_{i j} \frac{\partial^{2} \stackrel{2}{g}_{00}}{\partial x^{i} \partial x^{j}}-\left(\frac{\partial^{2} \stackrel{2}{g}_{00}}{\partial x^{i}}\right)^{2} \\
& -\frac{8 \pi G}{c_{l}^{4}}\left[\stackrel{2}{T^{00}}-2 \stackrel{2}{g}_{00} \stackrel{0}{T}^{00}\right] \\
\nabla^{2} \stackrel{2}{g}_{i 0} & =16 \pi G T^{i 0} \\
\nabla^{2} \stackrel{2}{g}_{i j} & =-\frac{8 \pi G}{c_{l}^{4}} \stackrel{0}{\delta}_{i j}^{0}
\end{array}\right\}
$$

From the first equation in (39) we find as expected

$$
\stackrel{2}{g}_{00}=-2 \phi
$$

where $\phi$ is the Newtonian potential defined by Poisson's equation

$$
\nabla^{2} \phi=\frac{8 \pi G}{c^{4}} T^{00}
$$

Also $\stackrel{2}{g}$ o0 must vanish at infinity, so the solution is

$$
\phi(x, t)=-\frac{G}{c^{4}} \int \frac{T^{00}\left(x^{\prime}, t\right)}{\left|x-x^{\prime}\right|} d^{3} x^{\prime}
$$

From the fourth equation in (39) we find that the solution for $\stackrel{2}{g}_{i j}$ that vanishes at infinity is

$$
\stackrel{2}{g}_{i i}=-2 \delta_{i j} \phi
$$

On the other hand, $\stackrel{3}{g}_{i 0}$ is a new vector potential $\varsigma$

$$
\stackrel{3}{g_{i 0}}=\varsigma_{i}
$$

and the solution of the third equation in (39) that vanishes at infinity is 


$$
\varsigma_{i}(x, t)=-\frac{4 G}{c^{4}} \int \frac{\stackrel{1}{T}^{i 0}\left(x^{\prime}, t\right)}{\left|x-x^{\prime}\right|} d^{3} x^{\prime}
$$

Finally, we may simplify the second equation in (39) by using (41), (42) and the identity

$$
\frac{\partial \phi}{\partial x^{i}} \frac{\partial \phi}{\partial x^{i}} \equiv \frac{1}{2} \nabla^{2} \phi^{2}-\phi \nabla^{2} \phi
$$

the result is

$$
\stackrel{4}{g}^{00}=-2 \phi^{2}-2 \psi
$$

where $\psi$ is a second potential

$$
\nabla^{2} \psi=\frac{\partial^{2} \phi}{\partial t^{2}}+\frac{4 \pi G}{c^{4}} \stackrel{2}{T} 00
$$

Again, $\stackrel{4}{g}_{00}$ must vanish at infinity, so the solution is

$$
\psi(x, t)=-\frac{G}{c^{4}} \int \frac{2^{0}{ }^{00}\left(x^{\prime}, t\right)}{\left|x-x^{\prime}\right|} d^{3} x^{\prime}
$$

To evaluate these potentials we need the proper time derivatives which can be obtained from the static Schwarzschild line element (in isotropic coordinates) for an observer at the position of $M_{1}$ or $M_{2}$.

$$
d \tau_{n}^{2}=g_{n 00} d t^{2}
$$

Using Misner, et al. [14] yields

$$
g_{n 00}=\left(1-\frac{G M_{n}}{2 c^{2} r_{n}}\right)^{2}\left(1+\frac{G M_{n}}{2 c^{2} r_{n}}\right)^{-2}
$$

To the order of the required accuracy we find

$$
\begin{aligned}
& \frac{d t}{d \tau_{1}}=1-\frac{2 G M_{2}}{c^{2} R} \\
& \frac{d t}{d \tau_{2}}=1-\frac{2 G M_{1}}{c^{2} R}
\end{aligned}
$$

The determinant of the metric tensor is given by

$$
\begin{gathered}
g=1-\stackrel{2}{g}+\stackrel{4}{g_{00}}+{ }_{00}=1-4 \phi \\
\frac{1}{\sqrt{g}}=1+2 \phi
\end{gathered}
$$

Substituting these all into the second potential $\psi$ yields

$$
\psi(x, t)=\frac{2 G^{2} M_{1} M_{2}}{c^{4} R}\left(\frac{1}{r_{1}}+\frac{1}{r_{2}}\right)
$$

Returning to the metric tensor components yields

$$
\left.\begin{array}{rl}
g_{00}= & -1+\frac{2 G M_{1}}{c^{2} r_{1}}+\frac{2 G M_{2}}{c^{2} r_{2}}+\left(\frac{2 G M_{1}}{c^{2} r_{1}}+\frac{2 G M_{2}}{c^{2} r_{2}}\right)^{2} \\
& -\frac{2 G^{2} M_{1} M_{2}}{c^{4} R}\left(\frac{1}{r_{1}}+\frac{1}{r_{2}}\right) \\
g_{i i}= & -1+\frac{2 G M_{1}}{c^{2} r_{1}}+\frac{2 G M_{2}}{c^{2} r_{2}}, g_{i 0}=0, \text { otherwise }
\end{array}\right\}
$$

\section{The Equations of Motion of Restricted Three-Body Problem}

The standard form of Euler-Lagrange equations read

$$
\frac{d}{d \tau}\left(\frac{\partial L}{\partial \dot{x}^{\alpha}}\right)-\frac{\partial L}{\partial x^{\alpha}}=0
$$

It can be shown that the geodesic equations of motion can be obtained from the Euler-Lagrange equations by defining the Lagrangian $L$ as follows (see Foster, and Nightingale, [15])

$$
L=\frac{1}{2} g_{\alpha \beta} \dot{x}^{\alpha} \dot{x}^{\beta}, \alpha, \beta=0,1,2,3
$$

where the dot denotes the derivatives with respect to proper time $\tau$, and $g_{\mu \gamma}$ are the components of the covariant metric tensor. Using the components of the metric tensor the Lagrangian, after some lengthy computations, can be constructed. Then evaluating the derivatives in EulerLagrange equations results in the equations of motion (61) and (62) in the following section.

\section{The Restricted Three-Body Problem Notations}

The well known Restricted Three-Body Problem, e.g. the Earth-Moon system, (from now on, RTBP) models the motion of an infinitesimal particle $\mathrm{P}$ under the gravitational attraction of two massive bodies, usually called primaries of masses $\left(M_{1}=1-\mu, M_{2}=\mu\right)$, under the following assumptions:

1) The particle is of infinitesimal mass that it does not affect the motion of the primaries,

2) The primaries are point masses that revolve in circular orbits around their common centre of mass.

It is usual to take a rotating reference frame with the origin at the centre of mass, and such that the two massive bodies are kept fixed on the $\xi$ axis, the $(\xi, \eta)$ plane is the plane of motion of the primaries, and the $\zeta$ axis is orthogonal to the $(\xi, \eta)$ plane. These coordinates are sometimes called synodical.

In the restricted three-body problem this must be transformed from the inertial $(x, y, z)$ to the rotating 
coordinate system $(\xi, \eta, \zeta)$ by using the transformation

$$
\begin{aligned}
\left(\begin{array}{l}
x \\
y \\
z
\end{array}\right)= & \left(\begin{array}{ccc}
\cos n t & -\sin n t & 0 \\
\sin n t & \cos n t & 0 \\
0 & 0 & 1
\end{array}\right)\left(\begin{array}{l}
\xi \\
\eta \\
\zeta
\end{array}\right) \quad \begin{array}{l}
\text { The primary coordinates on the } x \text {-axis }(-\mu, 0), \\
\text { 0) are kept fixed and the origin at the center of } \\
\text { Now denoting } x^{\alpha}, \alpha=1,2,3 \text { for } \xi, \eta, \zeta \text { in the } \\
\text { Lagrangian Equation (58), we obtain }
\end{array} \\
\ddot{\xi}= & 2 n \dot{\eta} \dot{t}+n^{2} \xi \dot{t}+n \eta \ddot{t}-G \dot{t}^{2}\left(\frac{(1-\mu)(\xi+1-\mu)}{r_{1}^{3}}+\frac{\mu(\xi-\mu)}{r_{2}^{3}}\right) \\
& +\frac{2 G}{c^{2}}\left\{\left(\frac{(1-\mu)[(\xi+1-\mu) \dot{\xi}+\eta \dot{\eta}]}{r_{1}^{3}}+\frac{\mu[(\xi-\mu) \dot{\xi}+\eta \dot{\eta}]}{r_{2}^{3}}\right)(\dot{\xi}-n \eta \dot{t})\right. \\
& +\left(\frac{1-\mu}{r_{1}}+\frac{\mu}{r_{2}}\right)\left\{-\ddot{\xi}+2 n \dot{\eta} \dot{t}+n \eta \ddot{t}+n^{2} \xi \dot{t}^{2}\right\}+G \dot{t}^{2}\left\{\left(\frac{1-\mu}{r_{1}}+\frac{\mu}{r_{2}}\right) \times\left(\frac{(1-\mu)(\xi+1-\mu)}{r_{1}^{3}}+\frac{\mu(\xi-\mu)}{r_{2}^{3}}\right)\right. \\
& \left.+\frac{1}{2}(1-\mu) \mu\left(\frac{(\xi+1-\mu)}{r_{1}^{3}}+\frac{(\xi-\mu)}{r_{2}^{3}}\right)\right\}-\left(\frac{(1-\mu)(\xi+1-\mu)}{2 r_{1}^{3}}+\frac{\mu(\xi-\mu)}{2 r_{2}^{3}}\right) \\
& \left.\left.\times\left(\dot{\xi}^{2}+\dot{\eta}^{2}+n^{2} \dot{t}^{2}\left(\xi^{2}+\eta^{2}\right)+2 n \dot{t}(\dot{\eta} \xi-\dot{\xi} \eta)\right)\right\}\right\} \\
\ddot{\eta}= & -2 n \dot{\xi} \dot{t}+n^{2} \eta \dot{t}+n \xi \ddot{t}-G \eta \dot{t}^{2}\left(\frac{(1-\mu)}{r_{1}^{3}}+\frac{\mu}{r_{2}^{3}}\right) \\
& +\frac{2 G}{c^{2}}\left\{\left(\frac{(1-\mu)[(\xi+1-\mu) \dot{\xi}+\eta \dot{\eta}]}{r_{1}^{3}}+\frac{\mu[(\xi-\mu) \dot{\xi}+\eta \dot{\eta}]}{r_{2}^{3}}\right)(\dot{\eta}-n \xi \dot{t})\right. \\
& +\left(\frac{1-\mu}{r_{1}}+\frac{\mu}{r_{2}}\right)\left(-\ddot{\eta}+2 n \ddot{\xi} \dot{t}+n \xi \ddot{t}+n^{2} \eta \dot{t}^{2}\right)+G \eta \dot{t}^{2}\left(\left(\frac{1-\mu}{r_{1}}+\frac{\mu}{r_{2}}\right)\left(\frac{(1-\mu)}{r_{1}^{3}}+\frac{\mu}{r_{2}^{3}}\right)+\frac{1}{2}(1-\mu) \mu\left(\frac{1}{r_{1}^{3}}+\frac{1}{r_{2}^{3}}\right)\right) \\
& \left.-\eta\left(\frac{(1-\mu)}{2 r_{1}^{3}}+\frac{\mu}{2 r_{2}^{3}}\right) \times\left(\dot{\xi}^{2}+\dot{\eta}^{2}+n^{2} \dot{t}^{2}\left(\xi^{2}+\eta^{2}\right)+2 n \dot{t}(\dot{\eta} \xi-\dot{\xi} \eta)\right)\right\}
\end{aligned}
$$

and

$$
\begin{aligned}
K= & \left\{\left\{1+\frac{2 G}{c^{2}}\left(\frac{1-\mu}{r_{1}}+\frac{\mu}{r_{2}}\right)^{2}\right\} \times n^{2}\left[(\dot{\eta} \xi-\dot{\xi} \eta)^{2}-\left(\xi^{2}+\eta^{2}\right)\left(\dot{\xi}^{2}+\dot{\eta}^{2}\right)\right]+\left\{1+\frac{2 G}{c^{2}}\left(\frac{1-\mu}{r_{1}}+\frac{\mu}{r_{2}}\right)\right\} \times\right. \\
& n^{2} c^{2}\left(\xi^{2}+\eta^{2}\right)+c^{4}\left\{1-\frac{2 G}{c^{2}}\left(\frac{1-\mu}{r_{1}}+\frac{\mu}{r_{2}}\right)+\frac{2 G}{c^{4}}\left(\frac{1-\mu}{r_{1}}+\frac{\mu}{r_{2}}\right)^{2}+\frac{2 G^{2} \mu(1-\mu)}{c^{4}(1-2 \mu)}\left(\frac{1}{r_{1}}+\frac{1}{r_{2}}\right)^{2}\right\} \\
& +c^{2}\left\{1-\frac{2 G}{c^{2}}\left(\frac{1-\mu}{r_{1}}+\frac{\mu}{r_{2}}\right)+\frac{2 G}{c^{4}}\left(\frac{1-\mu}{r_{1}}+\frac{\mu}{r_{2}}\right)^{2}+\frac{2 G^{2} \mu(1-\mu)}{c^{4}(1-2 \mu)}\left(\frac{1}{r_{1}}+\frac{1}{r_{2}}\right)^{2}\right\} \\
& \left.\times\left(1+\frac{2 G}{c^{2}}\left(\frac{1-\mu}{r_{1}}+\frac{\mu}{r_{2}}\right)\right)\left(\dot{\xi}^{2}+\dot{\eta}^{2}\right)\right)^{\frac{1}{2}}
\end{aligned}
$$


The coordinate time can be eliminated from the equations of motion by using Equation (57), the transformation (60) and the general relativistic line element

$$
\begin{aligned}
c^{2} d \tau^{2} & =d s^{2}=g_{\alpha \beta} d x^{\alpha \beta} \\
& =c^{2} d t^{2}-g_{11}\left(d x^{2}+d y^{2}\right)
\end{aligned}
$$

which leads to

$$
\begin{aligned}
\left(d x^{2}+d y^{2}\right)= & n^{2}\left(d \xi^{2}+d \eta^{2}\right) d t^{2}+\left(d \xi^{2}+d \eta^{2}\right) \\
& +2 n \xi d \eta d t-2 n \eta d \xi d t
\end{aligned}
$$

Thus the line element in the rotating coordinate system is

$$
\begin{aligned}
c^{2} d \tau^{2}= & c^{2} g_{00} d t^{2}-g_{00}\left(n^{2}\left(d \xi^{2}+d \eta^{2}\right) d t^{2}\right. \\
& +\left(d \xi^{2}+d \eta^{2}\right)+2 n \xi d \eta d t-2 n \eta d \xi d t
\end{aligned}
$$

Dividing by $d \tau^{2}$ allows an expression for $t$ to be obtained. This can then be used to eliminate $t$ from Equations (61) and (62) and after some algebraic manipulation, we get

$$
\left.\begin{array}{l}
\ddot{\xi}-2 n \dot{\eta}=\frac{\partial U}{\partial \xi}-\frac{d}{d t}\left(\frac{\partial U}{\partial \xi}\right) \\
\ddot{\eta}+2 n \dot{\xi}=\frac{\partial U}{\partial \eta}-\frac{d}{d t}\left(\frac{\partial U}{\partial \eta}\right)
\end{array}\right\}
$$

where $U$ is the potential-like function of the relativistic restricted three-body problem, which can be written as composed of two components, namely the potential of the classical restricted three-body problem $U_{c}$ and the relativistic correction $U_{r}$;

$$
U=U_{c}+U_{r}
$$

where $U_{c}$ and $U_{r}$ are given by

$$
U_{c}=\frac{r^{2}}{2}+\frac{1-\mu}{r_{1}}+\frac{\mu}{r_{2}}
$$

and

$$
\begin{aligned}
& U_{r}=\frac{r^{2}}{2 c^{2}}(\mu(1-\mu)-3)+\frac{1}{8 c^{2}}\left((\xi+\dot{\eta})^{2}\right. \\
& \left.+(\eta-\dot{\xi})^{2}\right)^{2}+\frac{3}{2 c^{2}}\left(\frac{1-\mu}{r_{1}}+\frac{\mu}{r_{2}}\right) \\
& \times\left((\xi+\dot{\eta})^{2}+(\eta-\dot{\xi})^{2}\right)-\frac{1}{2 c^{2}}\left(\frac{1-\mu}{r_{1}}+\frac{\mu}{r_{2}}\right)^{2} \\
& -\frac{\mu(1-\mu)}{2 c^{2}}\left\{\frac{1}{r_{1}}+\left(\frac{1}{r_{1}}-\frac{1}{r_{2}}\right)(1-3 \mu-7 \xi-8 \dot{\eta})\right. \\
& +\eta^{2}\left(\frac{\mu}{r_{1}^{3}}+\frac{1-\mu}{r_{2}^{3}}\right)
\end{aligned}
$$

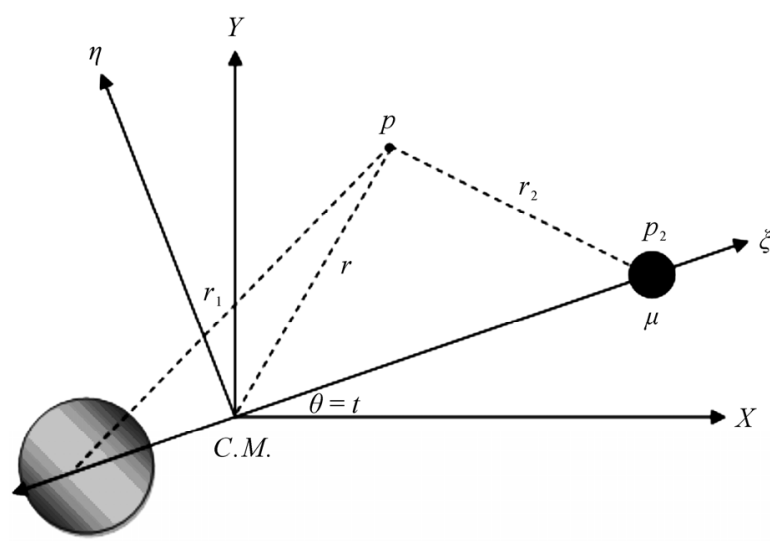

Figure 1. Inertial and rotating frames. The rotating coordinate system with coordinates $\xi$ and $\eta$ moves counterclockwise with unit angular velocity relative to the inertial frame with coordinates $X$ and $Y$.

with

$$
\left.\begin{array}{l}
n=1+\frac{1}{2 c^{2}}(\mu(1-\mu)-3), \\
r=\sqrt{\left(\xi^{2}+\eta^{2}\right)}, \\
r_{1}=\sqrt{(\xi+\mu)^{2}+\eta^{2}}, \\
r_{2}=\sqrt{(\xi+\mu-1)^{2}+\eta^{2}} .
\end{array}\right\}
$$

where the parameters $\left(r, r_{1}, r_{2}\right)$ are best illustrated in Figure 1.

\section{Conclusions}

An explicit form of the potential-like function of the relativistic restricted three-body problem is derived. Equations (65) to (68) represent the equations of motion of the relativistic three body problem in the PN formalism. These equations will be used in the subsequent works to evaluate the locations of the Lagrangian points, and therefore to investigate their linear stability.

\section{References}

[1] C. G. Jacobi, "Sur le Movement d'un Point et sur un cas Particulier du Probleme des trios Corps," Compte Rendus de l'Académie des Sciences, Vol. 3, 1836, p. 59.

[2] G. W. Hill, "Researches in the Lunar Theory," American Journal of Mathematics, Vol. 1, No. 1, 1878, pp. 5-26. doi:10.2307/2369430

[3] H. Poincaré, "Les Methodes Mouvelles de la Mecanique Celeste,” Vol. 3, Gauthier-Villars, Paris, 1892.

[4] G. D. Birkhoff, “The Restricted Problem of Three Bodies," Rendiconti del Circolo Matematico di Palermo, 
Vol. 39, 1915, pp. 265-334. doi:10.1007/BF03015982

[5] L. Euler, “Theoria Motuum Lumae,” Typis Academiae Imperialis Scientiarum, Petropoli, 1772.

[6] E. Krefetz, "Restricted Three-Body Problem in the PostNewtonian Approximation,” Astronomical Journal, Vol. 72, 1967, p. 471. doi:10.1086/110252

[7] G. Contopoulos, "In Memoriam D. Eginitis,” D. Kotsakis, Ed., Athens, 1976, p. 159.

[8] S. Weinberg, "Gravitation and Cosmology Principles and Applications of the General Theory of Relativity," Chapter 9, John Wiley \& Sons, New York, 1972.

[9] M. H. Soffel, "Relativity in Astrometry, Celestial Mechanics and Geodesy,” Springer-Verlag, Berlin, 1989, p. 173.

[10] V. A. Brumberg, "Relativistic Celestial Mechanics," Nauka Press (Science), Moscow, 1972.
[11] V. A. Brumberg, "Essential Relativistic Celestial Mechanics,” Adam Hilger, Ltd., New York, 1991.

[12] K. B. Bhatnagar and P. P. Hallan, "Existence and Stability of $L_{4,5}$ in the Relativistic Restricted Three Body Problem,” Celestial Mechanics, Vol. 69, No. 3, 1998, pp. 271281.

[13] F. W. Lucas, "Chaotic Amplification in the Relativistic Restricted Three-Body Problem,” Verlag der Zeitschrift für Naturforschung, Tübingen, Vol. 58a, No. 1, 2003, pp. 13-22.

[14] C. W. Misner, K. S. Thorne and J. A. Wheeler, "Gravitation,” Freeman and Company, San Francisco, 1973, p. 840.

[15] J. Foster and J. D. Nightingale, "A Short Course in General Relativity,” Springer, New York, 1995, p. 147. 